Gewichtung vorzudringen. Die angebotene zweieinhalbseitige „Theoriensynthese" (107 ff.) bleibt über einige formulierte Präferenzen für eine akteursorientierte, zwangsläufig auch eher kurzfristige und deskriptive Betrachtung hinaus relativ vage. Ähnliches gilt für den knappen „Ausblick“ (533) am Ende des Bandes. Auch dieser zitiert in erster Linie Diamond und Huntington, wobei die Schlussfolgerung, dass der Anteil der „liberalen“ an den „formalen“ Demokratien seit 1990 deutlich und beständig zurückgegangen sei (535), obwohl ihre absolute Zahl sich seitdem erheblich erhöht hat, in dieser Form wenig aussagekräftig ist. Gerade die Erscheinungsformen und Mängel der letzteren und die weiter wirkenden Ursachen (auch in den nicht behandelten Regionen) gilt es schärfer zu erfassen.

Über diese allgemeine Einschätzung hinaus seien auch noch einige konkrete Kritikpunkte angemerkt. Die von Huntington übernommene Periodisierung der „drei Wellen“, wenn hierunter auch gemeinsame Ursachen und eine kohärentere Theorie verstanden werden sollen, kann auch in der hier gewählten Darstellung nicht überzeugen. Nicht von ungefähr trennt der Verfasser daher die Darstellung der Prozesse in Südeuropa von denen in Osteuropa nach 1989. Seine "Typologie“ autokratischer Systeme (34 ff.) unterscheidet acht Formen autoritärer Herrschaftslegitimation, gelangt aber dann in enumerativ-deskriptiver Weise zu neun „Grundtypen“. Die Zuordnung der „Modernisierungstheorie“ (83 ff.) zu den "Systemtheorien " ist in dieser Form ebenfalls problematisch. Die vergleichende Darstellung der demokratischen Konsolidierung bzw. der Stagnation und des Scheiterns (485 ff.) kann bei vier a priori festgelegten Subtypen mit je einem konkreten Fall keine zusätzlichen analytischen, theoretisch fruchtbar zu machenden Erträge bringen.

Fazit: Ein für Überblicks- und Lehrzwecke in weiten Passagen gut geeigneter Band, der seine Stärke in den deskriptiven Teilen der Regionalanalysen und gewissen übergreifenden allgemeinen Klassifikationen hat, dessen theoretische Teile aber noch weiterer empirischer Ergänzung und analytischer Durchdringung bedürfen. Damit steht er aber, auch international, nicht allein. Es bleibt zu viel zu tun ...
Uhl, Manfred: Verfassungen in den politischen Systemtransformationen Osteuropas. Die postsozialistischen Verfassungsordnungen in der Russischen Föderation, Belarus und Lettland. Würzburg: Ergon Verlag 1999, 411 S., DM 75,--

Vorndran, Oliver: Die Entstehung der ukrainischen Verfassung. Berlin: Duncker \& Humblot 2000, 355 S., DM 132,-.

\section{Florian Grotz}

Mit dem Zusammenbruch des realen Sozialismus sind die Verfassungen der osteuropäischen Staaten gleichsam über Nacht zu einem zentralen Gegenstand politikwissenschaftlicher Analyse geworden. Während verfassungsrechtliche Regelungen unter der kommunistischen Herrschaft so gut wie keine realpolitischen Auswirkungen gezeitigt haben, scheint nun das genaue Gegenteil der Fall: In den jungen Demokratien waren die neuen Verfassungen nicht nur politisch stark umstritten; auch die politikwissenschaftliche Transformationsforschung hat der Form politischer Institutionen großen Einfluss auf die demokratische Konsolidierung zuerkannt und vor diesem Hintergrund Vorzüge und Nachteile bestimmter Verfassungsarrangements intensiv debattiert.

Angesichts der theoretischen wie politischpraktischen Bedeutsamkeit post-sozialistischer Verfassungen verwundert es kaum, dass sich mittlerweile eine wachsende Zahl von Publikationen zu diesem Thema findet. Von einem generellen Defizit an Verfassungsanalysen kann in der "neuen Osteuropaforschung" wahrlich keine Rede mehr sein. Allerdings gibt es in der Forschung zu den politischen Auswirkungen post-sozialistischer Verfassungsarrangements wie auch hinsichtlich der Rekonstruktion osteuropäischer Verfassungsgebungsprozesse noch etliche „weiße Felder“. Beide hier anzuzeigenden Studien haben sich jeweils die Behebung eines solchen Forschungsdesiderats vorgenommen - mit unterschiedlichem Erfolg.

Manfred Uhl hat sich in seiner Augsburger Dissertation mit der „Verfassungstransformation" in Russland, Belarus und Lettland auseinandergesetzt. Konkret geht es dabei um die Frage, „welche Ordnungsprinzipien [...] in die Verfassungen implementiert [wurden] und welche Ausprägungen [...] diese erfahren [haben]" (22 f.). Die eigentliche Leistung seiner 